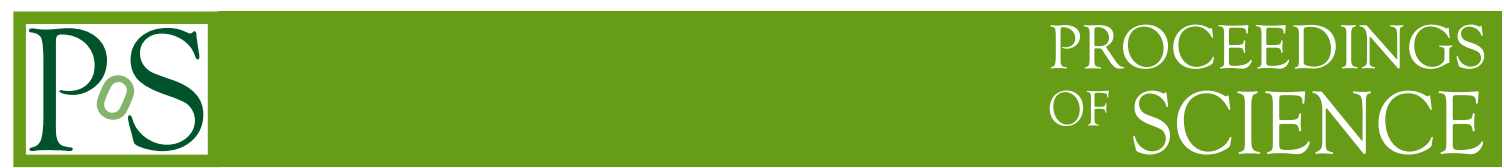

\title{
NLO QCD corrections to Higgs boson pair production
}

\author{
Julien Baglio, ${ }^{a}$ Francisco Campanario, ${ }^{b, c}$ Seraina Glaus,,${ }^{c, d, *}$ Milada Margarete \\ Mühlleitner, ${ }^{c}$ Jonathan Ronca $^{b}$ and Michael Spira ${ }^{e}$ \\ ${ }^{a}$ Theoretical Physics Department, CERN, \\ Esplanade des Particules 1, 1217 Meyrin, Switzerland \\ ${ }^{b}$ Theory Division, IFIC, University of Valencia-CSIC, \\ Parque Científico, C/Catedrático José Beltrán, 2, E-46980 Paterna, Spain \\ ${ }^{c}$ Institute for Theoretical Physics, Karlsruhe Institute of Technology \\ Wolfgang-Gaede-Straße 1, 76128 Karlsruhe, Germany \\ ${ }^{d}$ Institute for Nuclear Physics, Karlsruhe Institute of Technology, \\ Hermann-von-Helmholtz-Platz 1, 76344 Karlsruhe, Germany \\ ${ }^{e}$ Laboratory for Particle Physics, Paul Scherrer Institute, \\ Forschungsstrasse 111, 5232 Villigen, Switzerland \\ E-mail: julien.baglio@cern.ch, Francisco.Campanario@ific.uv.es, \\ seraina.glaus@kit.edu, milada.muehlleitner@kit.edu, Jonathan.Ronca@uv.es, \\ Michael.Spira@psi.ch
}

In this contribution the next-to-leading (NLO) QCD corrections to Higgs boson pair production are discussed. A brief sketch of the calculation is given. The differential cross section as a function of the invariant Higgs pair mass and the total hadronic cross section are presented. Furthermore, the uncertainties not only from the renormalisation and factorisation scales but also the uncertainties due to the scheme-and-scale choice of the top mass are shown. In addition, the effects of varying the Higgs self-coupling strength on the cross section are investigated.

The Eighth Annual Conference on Large Hadron Collider Physics-LHCP2020

25-30 May, 2020

online

${ }^{*}$ Speaker 


\section{Introduction}

Since the detection of a Higgs boson in 2012 [1, 2] many of its properties have been determined and are in agreement with the Standard Model (SM) predictions [3-8]. However, the Higgs selfcoupling strength $\lambda_{H^{3}}$ is still unknown. It is of particular interest since it gives access to the Higgs potential which is directly related to the electroweak symmetry breaking mechanism. Higgs pair production needs to be considered to directly probe the Higgs self-coupling strength. The dominant production channel at the LHC for Higgs pair production is gluon fusion $g g \rightarrow H H$, a loop-induced process where the top quark in the loops provides the dominant contribution [9, 10]. Its cross section is about three orders of magnitude smaller than the gluon-fusion cross section of single Higgs production [11]. For gluon fusion the relative uncertainty of the cross section is about the same as the impact of the same relative variation of the Higgs self-coupling strength around its SM value. Therefore higher-order corrections to the cross section are required in order to reduce the uncertainty in the Higgs self-couplings strength measurement.

In a first step, the next-to-leading-order (NLO) QCD corrections have been calculated in the large top mass limit (HTL) where corrections of around $100 \%$ have been obtained [12]. Later, a large top mass expansion has been added which leads to additional $10 \%$ NLO mass corrections [13, 14]. These have been confirmed by calculating the real corrections with the full mass dependence [15]. Recently, two independent group have calculated the NLO QCD corrections including the full top mass dependence using two different approaches [16-20]. Thereby, NLO mass effects of $-15 \%$ in addition to the HTL result have been obtained. This result has been supported by various expansion and extrapolation methods [21-23].

\section{Calculation of the NLO QCD corrections}
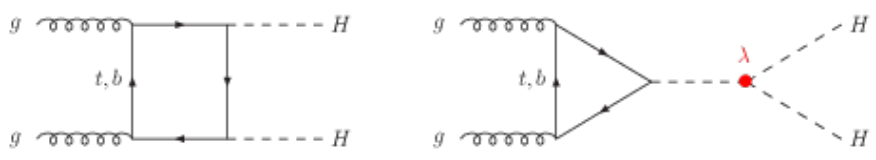

Figure 1: Generic diagrams for Higgs boson pair production at LO where the contribution of the trilinear Higgs coupling is highlighted in red.

At leading order (LO) gluon fusion consists of diagrams involving the Higgs self-coupling and of such involving the Yukawa couplings only (Figure 1). Diagrams with no Higgs self-coupling will be designated as box diagrams and the rest as triangular diagrams. For the virtual corrections the triangle diagrams have been constructed from the known results of single Higgs production by adding the Higgs vertex at the level of the matrix elements. For the one-particle reducible diagrams the results of $H \rightarrow Z \gamma$ have been used by adjusting the kinematics and couplings accordingly. The challenging part was the calculation of the virtual corrections of the box diagrams. Here a diagrammatic approach has been chosen and no reduction to master integrals has been performed. The ultraviolet divergences of the matrix elements were extracted using endpoint subtraction. For the infrared and collinear divergences a similar subtraction but with more involved subtraction terms has been used. To cope with the numerical instabilities above the virtual thresholds integration 


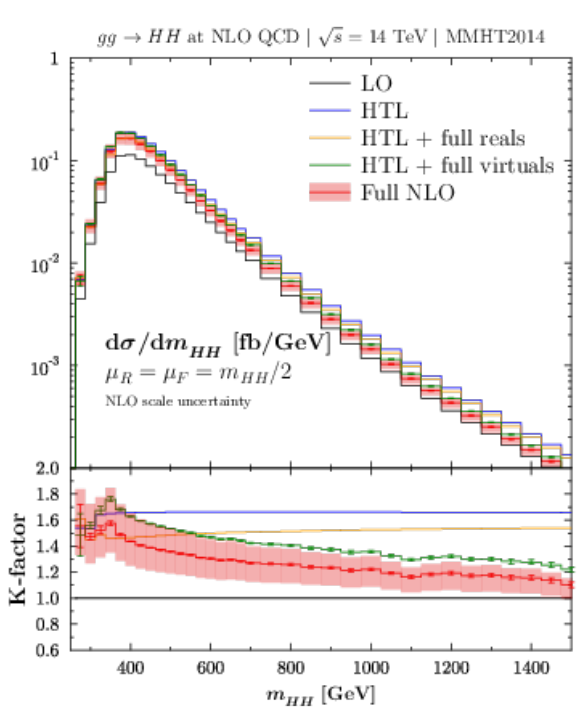

Figure 2: The upper panel displays the invariant Higgs pair mass distribution at 14 $\mathrm{TeV}$ and the lower panel the K-factor as a function of the invariant Higgs pair mass.

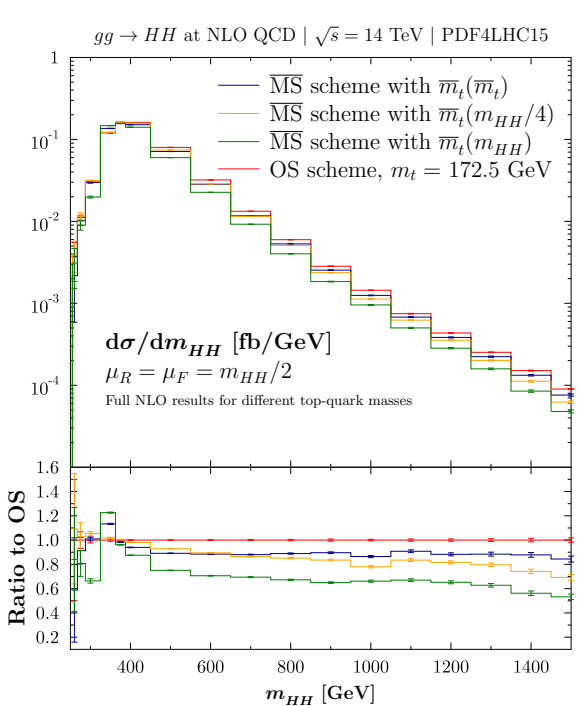

Figure 3: In the upper panel the differential NLO distributions in $m_{H H}$ for different scheme and scale choices of $m_{t}$ are depicted and in the lower panel the ratios of them to the one with the on-shell top mass.

by parts over one of the Feynman parameters were applied. The integration over the six Feynman parameters and one of the phase space variable was performed using Vegas [24] leading to the virtual part of the differential cross section in the invariant Higgs pair mass, $m_{H H}=Q$. The strong coupling $\alpha_{s}$ was renormalized in the $\overline{M S}$-scheme with five active flavours and the top mass $m_{t}$ in the on-shell scheme. Further instabilities were prevented by using a Richardson extrapolation. For the real corrections the matrix elements were generated with FeynArts [25] and FormCalc [26].

\section{Results}

The numerical results are presented for a centre-of-mass energy of $14 \mathrm{TeV}$, a Higgs mass $m_{H}=125 \mathrm{GeV}$ and a top mass $m_{t}=172.5 \mathrm{GeV}$. For the NLO PDF sets we used MMHT2014 [27] and PDF4LHC15 [28]. We have chosen a grid from $Q=250 \mathrm{GeV}$ to $1500 \mathrm{GeV}$ resulting in the invariant double-Higgs mass distribution in Figure 2. In red the NLO QCD corrections with the full mass dependence are shown. The error band displays the uncertainties due to the factorisation and renormalisation scale choice. The black curve represents the LO result, the blue curve the HTL result, the yellow the HTL supplemented by the full real corrections and the green one the HTL with the full virtual corrections. In the lower plot of Figure 2 it is visible that for low invariant Higgs pair masses $m_{H H}$ all the curves are of the same magnitude. However, for larger ones the green and the red curves differ a lot from the blue and the yellow curves. This supports the importance of the inclusion of the mass effects, especially the ones from the virtual corrections.

The numerical integration over $Q$ leads to the total hadronic cross section. For this purpose a combination of the trapezoidal method and Richardson extrapolation has been applied. For a 
centre-of-mass energy of $14 \mathrm{TeV}$ a cross section $\sigma=32.81(7)_{-12.5 \%}^{+13.5 \%} \mathrm{fb}$ has been obtained. The given errors are the uncertainties due to the factorisation and renormalisation scale choice.

In addition, the uncertainty related to the scheme-and-scale choice of the virtual intrinsic top mass has been calculated for the first time. For this purpose the differential cross section has been determined bin by bin for the $m_{t}$ in the on-shell scheme and in the $\overline{M S}$-schemes at the scale $\mu_{t}$ where we varied this scale in the range between $Q / 4$ and $Q$. The following results have been obtained,

$$
\begin{aligned}
& \left.\frac{\mathrm{d} \sigma(\mathrm{gg} \rightarrow \mathrm{HH})}{\mathrm{dQ}}\right|_{Q=300 \mathrm{GeV}}=0.02978(7)_{-34 \%}^{+6 \%} \mathrm{fb} / \mathrm{GeV}, \\
& \left.\frac{\mathrm{d} \sigma(\mathrm{gg} \rightarrow \mathrm{HH})}{\mathrm{dQ}}\right|_{Q=400 \mathrm{GeV}}=0.1609(4)_{-13 \%}^{+0 \%} \mathrm{fb} / \mathrm{GeV}, \\
& \left.\frac{\mathrm{d} \sigma(\mathrm{gg} \rightarrow \mathrm{HH})}{\mathrm{dQ}}\right|_{Q=600 \mathrm{GeV}}=0.03204(9)_{-30 \%}^{+0 \%} \mathrm{fb} / \mathrm{GeV}, \\
& \left.\frac{\mathrm{d} \sigma(\mathrm{gg} \rightarrow \mathrm{HH})}{\mathrm{dQ}}\right|_{Q=1200 \mathrm{GeV}}=0.000435(4)_{-35 \%}^{+0 \%} \mathrm{fb} / \mathrm{GeV}
\end{aligned}
$$

In Figure 3 the NLO differential cross sections in $m_{H H}$ for different scale-and-scheme choices of $m_{t}$ are depicted. The lower panel shows the ratios of the differential cross sections to the one with the on-shell top mass. The scale-and-scheme dependence of the top mass causes sizeable variations of the NLO cross section and cannot be neglected. Going to large $Q$-values the maximum is given by the on-shell scheme and the minimum by the $\overline{M S}$-scheme with $\bar{m}_{t}(Q)$. The resulting uncertainties are about a factor two smaller than for the LO result. For the uncertainty of the total hadronic cross section the envelope for each bin has been taken and then integrated over $Q$. For a centre of mass energy of $14 \mathrm{TeV}$ we obtained $\sigma=32.81(7)_{-18 \%}^{+4 \%} \mathrm{fb}$.

Furthermore, we investigated how the NLO cross section varies with modified Higgs selfcoupling strength. All the other couplings have been kept at their SM values. It has been observed that the NLO mass effects shift the minimum of the total cross section from 2.4 to 2.3 times the SM value of the Higgs self-coupling. This shift is mainly caused by the NLO mass effects of the real corrections. Except for the regions where destructive interference between triangle and box diagrams occurs, the NLO mass effects of the total cross section increase with higher centre-of-mass energies.

\section{Conclusions}

In this contribution, it is briefly explained how the NLO QCD corrections to Higgs boson pair production have been calculated. The differential cross section in $m_{H H}$ and the total hadronic cross section have been presented. The comparison of the invariant Higgs pair mass distribution in the HTL and of the full mass calculation has shown that the mass effects cannot be neglected for large $m_{H H}$. In addition to the uncertainties due to the renormalisation and factorisation scale dependence, for the first time also the uncertainties due to the scale-and-scheme choice of the top mass have been presented. They are of the same size as the renormalisation and factorisation scale dependence and thus cannot be neglected. Furthermore, it has been shown that the variation of $\lambda_{H^{3}}$ shifts the minimum of the Higgs boson pair production cross section from 2.4 to 2.3 times the SM value of the Higgs self-coupling. 


\section{References}

[1] ATLAS collaboration, Observation of a new particle in the search for the Standard Model Higgs boson with the ATLAS detector at the LHC, Phys. Lett. B716 (2012) 1 [1207 .7214].

[2] CMS collaboration, Observation of a new boson at a mass of $125 \mathrm{GeV}$ with the CMS experiment at the LHC, Phys. Lett. B716 (2012) 30 [1207.7235].

[3] P. W. Higgs, Broken symmetries, massless particles and gauge fields, Phys. Lett. 12 (1964) 132.

[4] P. W. Higgs, Broken Symmetries and the Masses of Gauge Bosons, Phys. Rev. Lett. 13 (1964) 508.

[5] P. W. Higgs, Spontaneous Symmetry Breakdown without Massless Bosons, Phys. Rev. 145 (1966) 1156.

[6] F. Englert and R. Brout, Broken Symmetry and the Mass of Gauge Vector Mesons, Phys. Rev. Lett. 13 (1964) 321.

[7] G. S. Guralnik, C. R. Hagen and T. W. B. Kibble, Global Conservation Laws and Massless Particles, Phys. Rev. Lett. 13 (1964) 585.

[8] T. W. B. Kibble, Symmetry breaking in nonAbelian gauge theories, Phys. Rev. 155 (1967) 1554.

[9] E. N. Glover and J. van der Bij, Higgs boson pair production via gluon fusion, Nucl. Phys. B309 (1988) 282.

[10] T. Plehn, M. Spira and P. M. Zerwas, Pair production of neutral Higgs particles in gluongluon collisions, Nucl. Phys. B479 (1996) 46 [hep-ph/9603205]. [Erratum: Nucl. Phys. B531 (1998) 655].

[11] J. Baglio, A. Djouadi, R. Gröber, M. M. Mühlleitner, J. Quevillon and M. Spira, The measurement of the Higgs self-coupling at the LHC: theoretical status, JHEP 1304 (2013) 151.

[12] S. Dawson, S. Dittmaier and M. Spira, Neutral Higgs boson pair production at hadron colliders: QCD corrections, Phys. Rev. D58 (1998) 115012 [hep-ph/9805244].

[13] J. Grigo, J. Hoff and M. Steinhauser, Higgs boson pair production: top quark mass effects at NLO and NNLO, Nucl. Phys. B900 (2015) 412 [1508.00909].

[14] J. Grigo, J. Hoff, K. Melnikov and M. Steinhauser, On the Higgs boson pair production at the LHC, Nucl. Phys. B875 (2013) 1 [1305 . 7340].

[15] R. Frederix, S. Frixione, V. Hirschi, F. Maltoni, O. Mattelaer et al., Higgs pair production at the LHC with NLO and parton-shower effects, Phys. Lett. B732 (2014) 142 [1401.7340]. 
[16] S. Borowka, N. Greiner, G. Heinrich, S. Jones, M. Kerner, J. Schlenk et al., Higgs Boson Pair Production in Gluon Fusion at Next-to-Leading Order with Full Top-Quark Mass Dependence, Phys. Rev. Lett. 117 (2016) 012001 [1604.06447]. [Erratum: Phys. Rev. Lett. 117 (2016) 079901].

[17] S. Borowka, N. Greiner, G. Heinrich, S. P. Jones, M. Kerner, J. Schlenk et al., Full top quark mass dependence in Higgs boson pair production at NLO, JHEP 10 (2016) 107 [1608 . 04798].

[18] J. Baglio, F. Campanario, S. Glaus, M. Mühlleitner, M. Spira and J. Streicher, Gluon fusion into Higgs pairs at NLO QCD and the top mass scheme, Eur. Phys. J. C79 (2019) 459 [1811.05692].

[19] J. Baglio, F. Campanario, S. Glaus, M. Mühlleitner, J. Ronca, M. Spira and J. Streicher, Higgs-Pair Production via Gluon Fusion at Hadron Colliders: NLO QCD Corrections, JHEP 04 (2020), 181 doi:10.1007/JHEP04(2020)181 [2003. 03227]

[20] J. Baglio, F. Campanario, S. Glaus, M. Mühlleitner, J. Ronca and M. Spira, $g g \rightarrow H H$ : Combined Uncertainties, [2008.11626]

[21] R. Gröber, A. Maier and T. Rauh, Reconstruction of top-quark mass effects in Higgs pair production and other gluon-fusion processes, JHEP 03 (2018) 020 [1709. 07799].

[22] R. Bonciani, G. Degrassi, P. P. Giardino and R. Gröber, Analytical Method for Next-toLeading-Order QCD Corrections to Double-Higgs Production, Phys. Rev. Lett. 121 (2018) 162003 [1806. 11564].

[23] J. Davies, G. Mishima, M. Steinhauser and D. Wellmann, Double Higgs boson production at NLO in the high-energy limit: complete analytic results, JHEP 01 (2019) 176 [1811.05489].

[24] G. P. Lepage, Vegas: An adaptive multidimensional Integration Program, CLNS-80/447 (1980) .

[25] T. Hahn, Generating Feynman diagrams and amplitudes with FeynArts 3, Comput. Phys. Commun. 140 (2001) 418 [hep-ph/0012260].

[26] T. Hahn and M. Perez-Victoria, Automatized one loop calculations in four-dimensions and D-dimensions, Comput. Phys. Commun. 118 (1999) 153 [hep-ph/9807565].

[27] L. A. Harland-Lang, A. D. Martin, P. Motylinski and R. S. Thorne, Parton distributions in the LHC era: MMHT 2014 PDFs, Eur. Phys. J. C75 (2015) 204 [1412 . 3989].

[28] J. Butterworth et al., PDF4LHC recommendations for LHC Run II, J. Phys. G43 (2016) $023001[1510.03865]$. 